\title{
HFDA: Hybrid Face Detection Algorithm for Analyzing of Biometric Application
}

\author{
Surbhi Choudhary \\ Research Scholar, \\ Department of Computer Science \\ Vindhya Institute of \\ Technology \& Science, \\ Indore (M.P.)
}

\author{
Ashish Tiwari \\ Asst. Prof. \& Head, \\ Department of CSE \& IT \\ Vindhya Institute of \\ Technology \& Science, \\ Indore (M.P.)
}

\begin{abstract}
Edges characterize boundaries and are therefore a problem of fundamental importance in image processing. Image Edge detection significantly reduces the amount of data and filters out useless information, while preserving the important structural properties in an image. Since edge detection is in the forefront of image processing for object detection, it is crucial to have a good understanding of edge detection algorithms. In this age of era, different authentication and authorization is required for security perspective. So in this concern for verifying or identify a person in the digital image, features extracted from the digital image are compared with features of the images in the facial database. In this paper, we have been implemented HFDA i.e. Hybrid Face Detection Algorithm using canny filter and LDA approach that enhance figures are found to be better than some of the work reported in literature.
\end{abstract}

\section{Keywords}

Face Recognition, Feature selection, LDA, PCA, Face Data, Computer Vision, Authentication, Canny Edge Filter.

\section{INTRODUCTION}

A wide variety of systems require reliable personal recognition schemes to either confirm or determine the identity of an individual requesting their services. The purpose of such schemes is to ensure that the rendered services are accessed only by a legitimate user, and not anyone else. Humans often use faces to recognize individuals and advancements in computing capability over the past few decades now enable similar recognitions automatically. Humans have the ability to recognize faces easily and effortlessly but in the area of image analysis and computer vision it remained as a difficult problem on which many years of research is going on. It is often useful to have a machine perform pattern recognition. In particular, machines which can read face images are very cost effective. The domain of machine learning and data analysis is frequently used in various real world applications. Some of them are used for providing ease in reducing the amount of data during intelligence system development and some of them are used for directly producing the outcomes to the applications. Among them the recognition and classification is a classical issue of machine learning. A machine that reads passenger passports can process many more passports than a human being in the same time [1].

\subsection{Biometric System}

A biometric system is essentially a pattern recognition system that operates by acquiring biometric data from an individual, extracting a feature set from the acquired data, and comparing this feature set against the template set in the database. Humans recognize each other according to their various characteristics for ages. We recognize others by their face when we meet them and by their voice as we speak to them. Identity verification (authentication) in computer systems has been traditionally based on something that one has (key, magnetic or chip card) or one knows (PIN, password). Things like keys or cards, however, tend to get stolen or lost and passwords are often forgotten or disclosed. To achieve more reliable verification or identification we should use something that really characterizes the given person [2].

Biometric systems are vulnerable to two types of failures: a false-positive, in which a system falsely identifies an imposter as the valid user, and a false-negative, in which the system fails to make a match between a valid user and the stored template. Because no single identifier is fool-proof, using more than one method, such as a biometric measure in addition to a personal identification number, can enhance security [3].

\subsection{Face Recognition}

Face recognition is a biometric technique used for surveillance purposes such as search for wanted criminals, suspected terrorists, and missing children. The term face recognition refers to identifying, by computational algorithms, an unknown face image. This operation can be done by comparing the unknown face with the faces stored in database. Face recognition has three stages a) face location detection b) feature extraction c) facial image classification [4]. Face recognition (FR) has emerged as one of the most extensively studied research topics that spans multiple disciplines such as pattern recognition, signal processing and computer vision. This is due to its numerous important applications in identity authentication, security access control, intelligent human-computer interaction, and automatic indexing of image and video databases [5]. Face is the index of mind. It is a complex multidimensional structure and needs a good computing technique for recognition. While using automatic system for face recognition, computers are easily confused by changes in illumination, variation in poses and change in angles of faces. A numerous techniques are being used for security and authentication purposes which includes areas in detective agencies and military purpose [6].

The face recognition techniques mainly work in three steps [7].

\section{Face Detection}

2. Feature Extraction

3. Face Recognition. 


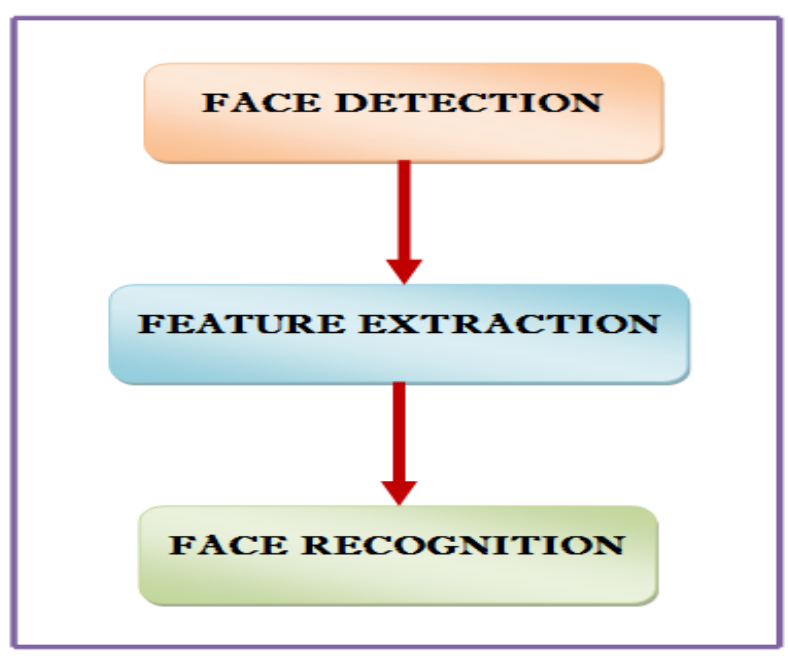

Figure 1: Face detection and Recognition Overview

\section{Face Detection}

Face detection can be regarded as a specific case of objectclass detection. In object-class detection, the task is to find the locations and sizes of all objects in an image that belong to a given class. Face detection can be regarded as a more general case of face localization. In face localization, the task is to find the locations and sizes of a known number of faces (usually one). In face detection, one does not have this additional information.

\section{Feature Extraction}

The In pattern recognition and in image processing, feature extraction is a special form of dimensionality reduction. When the input data to an algorithm is too large to be processed and it is suspected to be notoriously redundant then the input data will be transformed into a reduced representation set of features. After the FD step, human-face patches are extracted from images. Directly using these patches for FR have some disadvantages, first, each patch usually contains over 1000 pixels, which are too large to build a robust recognition system. Second, face patches may be taken from different camera alignments, with different face expressions, illuminations, and may suffer from occlusion and clutter. To overcome these drawbacks, feature extractions are performed to do information packing, dimension reduction, salience extraction, and noise cleaning. After this step, a face patch is usually transformed into a vector with fixed dimension or a set of fiducially points and their corresponding locations. Transforming the input data into the set of features is called feature extraction [8].

\section{Face Recognition}

A facial recognition system is a computer application for automatically identifying or verifying a person from a digital image or a video frame from a video source. One of the ways to do this is by comparing selected facial features from the image and a facial database. It is typically used in security systems and can be compared to other biometrics such as fingerprint or eye iris recognition systems. Among the different biometric techniques, facial recognition may not be the most reliable and efficient. However, one key advantage is that it does not require aid (or consent) from the test subject. Properly designed systems installed in airports, multiplexes, and other public places can identify individuals among the crowd [9].

\section{LITERATURE SURVEY}

In this paper Deepak et al. [10] propose a method to detect human faces in color images. Many existing systems use a window-based classifier that scans the entire image for the presence of the human face and such systems suffers from scale variation, pose variation, illumination changes, etc. Here, we propose a lighting insensitive face detection method based upon the edge and skin tone information of the input color image. First, image enhancement is performed, especially if the image is acquired from an unconstrained illumination condition. Next, skin segmentation in $\mathrm{YCbCr}$ and RGB space is conducted. The result of skin segmentation is refined using the skin tone percentage index method. The edges of the input image are combined with the skin tone image to separate all non-face regions from candidate faces. Candidate verification using primitive shape features of the face is applied to decide which of the candidate regions corresponds to a face. The advantage of the proposed method is that it can detect faces that are of different sizes, in different poses, and that are making different expressions under unconstrained illumination conditions.

Edge detection is very important terminology in image processing and for computer vision. Edge detection is in the forefront of image processing for object detection, so it is crucial to have a good understanding of edge detection operators. In the present study Pinaki Pratim Acharjya et al. [11], have comparative analyses of different edge detection operators in image processing are presented. It has been observed from the present study that the performance of canny edge detection operator is much better then Sobel, Roberts, Prewitt, Zero crossing and LoG (Laplacian of Gaussian) in respect to the image appearance and object boundary localization. The software tool that has been used is MATLAB.

Face recognition is one of the most challenging tasks in the field of image processing. This paper presents an optimized edge detection algorithm for the task of face recognition. In this method, M Sudarshan et al. [12] present gradient based filter using a wide convolution kernel is applied on the image to extract the edges. Later a thinning algorithm optimized for the wide convolution kernel is applied on the extracted edges. The advantages of this method over other gradient based methods is its ability to find the missing edges more successfully and boost the significant edges of the overall facial contour.

Since edge detection is in the forefront of image processing for object detection, it is crucial to have a good understanding of edge detection algorithms. In this paper, Mohsen Sharifi et al. [13] introduces a new classification of most important and commonly used edge detection algorithms, namely ISEF, Canny, Marr-Hildreth, Sobel, Kirsch, Lapla1 and Lapla2. Five categories are included in this classification, and then advantages and disadvantages of some available algorithms within this category are discussed. A representative group containing the above seven algorithms are the implemented in $\mathrm{C}++$ and compared subjectively, using 30 images out of 100 images. Two sets of images resulting from the application of those algorithms are then presented. It is shown that under noisy conditions, ISEF, Canny, Marr-Hildreth, Kirsch, Sobel, Lapla2, Lapla1 exhibit better performance, respectively.

Edge can be defined as discontinuities in image intensity from one pixel to another. Edge detection is one of the most fundamental algorithms in digital image processing. The Canny algorithm computes the higher and lower thresholds 
for edge detection based on the entire image statistics, which prevents the processing of blocks independent of each other. In this paper Poonam S. Deokar et al. [14] propose canny algorithm that is used as a standard from many years. However, this is a time consuming algorithm. Here a new approach is presented to calculate gradient using approximate method which reduces the latency and resource utilization. The proposed algorithm is implemented using FPGA.

\section{PROPOSED SYSTEM}

The proposed system is designed in two different modules; where are LDA and LDA Canny Edge Filter in which training and testing perform in both modules. Here in training module system accepts user data in form of Image and calculate features. In testing phase system accepts user query in form of image and system produces results and performance of results are listed. The basic overview of the system is given below.

\subsection{Methodology}

The working of the proposed HFDA algorithm is given and explained in details in this section. To understand the core concept of the proposed methodology by using given figure description:

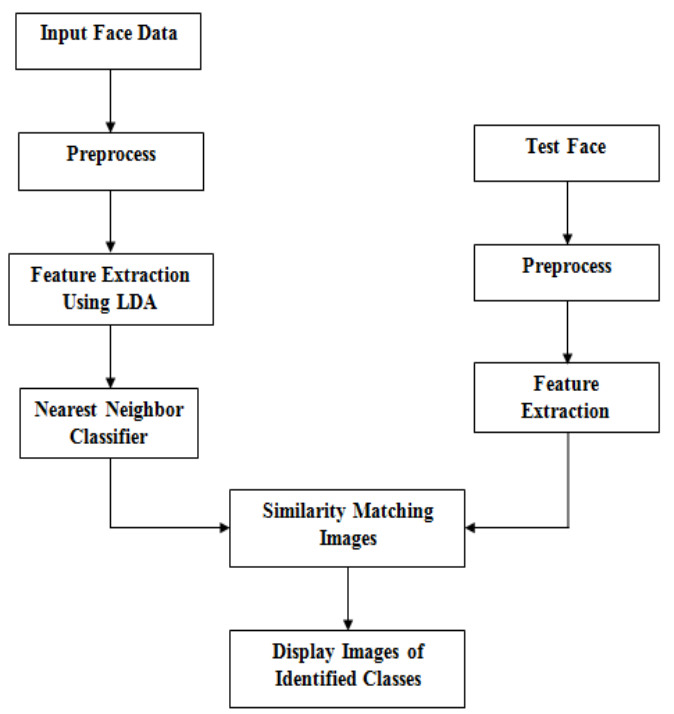

Figure 2: Basic LDA Working

The figure 2 depicts basic LDA Process which is identified matching images of the input faces. In this flow chart load the input data face to this method according their availability of images in database. Furthermore, on this preprocess the face data by which we get the important and selected feature of the image. In preprocess, generate by default pixel value of full image but we have to require only those feature on which apply LDA algorithm to extract image features. On this extracted features, to classify classes on basis of this features use nearest neighbor classifier KNN classifier is best suited for classifying persons based on their images due to its lesser execution time and better accuracy than other commonly used methods. The k-nearest neighbor algorithm (k-NN) is a method for classifying objects based on closest training examples in the feature space.

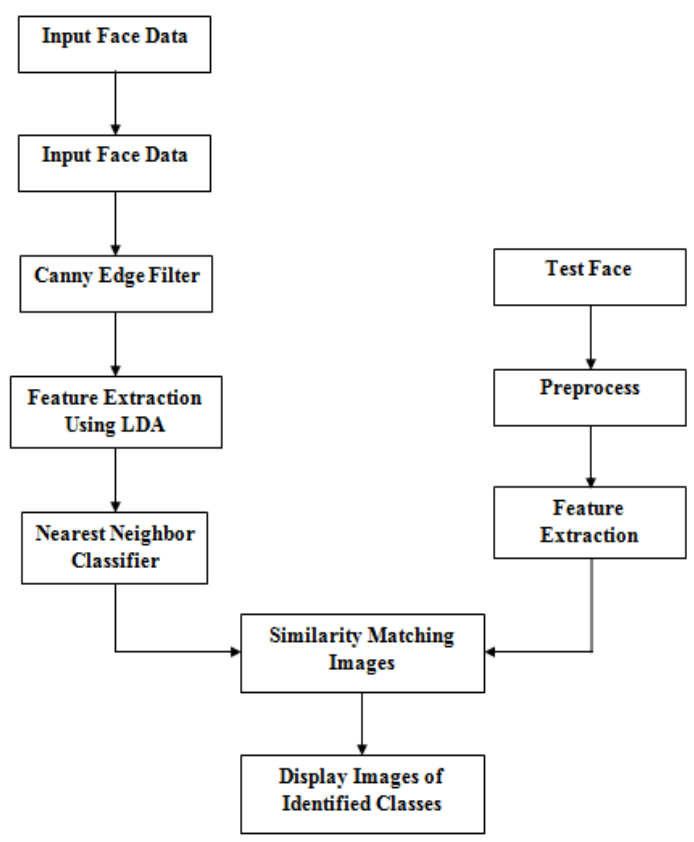

Figure 3: Proposed Hybrid Face Recognition flow

Similarly in figure 3proposed HFDA, the difference is that in both methods of hybridization that combine two method. Here on input face data we apply canny edge filter algorithm and for extracting feature and on this feature also apply LDA algorithm and finally we get optimal extracted features of this input image. Likewise, NN classify the different classes of similar attribute basis. On the other hand, load test faces for testing process, and preprocess this image and extract to get feature. So this extracted data or training phase and training phase to match similar type of image matching on basis of their features. In last, result image is displayed as output image of identified classes.

\subsection{Proposed Algorithm}

The given section provides the detailed understanding about the proposed algorithm face recognition system.

Summarizing the LDA approach in 5 steps

Listed below are the 5 general steps for performing a linear discriminant analysis; we will explore them in more detail in the following sections.

- Compute the -dimensional mean vectors for the different classes from the dataset.

- Compute the scatter matrices (in-between-class and within-class scatter matrix).

- Compute the eigenvectors and corresponding eigenvalues for the scatter matrices.

- Sort the eigenvectors by decreasing eigenvalues and choose eigenvectors with the largest eigenvalues to form a dimensional matrix (where every column represents an eigenvector).

- Use this eigenvector matrix to transform the samples onto the new subspace. This can be summarized by the matrix multiplication: (where is a dimensional matrix representing the nn samples, and are the transformed dimensional samples in the new subspace). 


\section{Canny Edge Detection}

The purpose of edge detection in general is to significantly reduce the amount of data in an image, while preserving the structural properties to be used for further image processing.

The algorithm runs in 5 separate steps:

- Smoothing: Blurring of the image to remove noise.

- Finding Gradients: The edges should be marked where the gradients of the image has large magnitudes.

- Non-Maximum Suppression: Only local maxima should be marked as edges.

- Double Thresholding: Potential edges are determined by thresholding.

- Edge Tracking by Hysteresis: Final edges are determined by suppressing all edges that are not connected to a very certain (strong) edge.

\section{RESULT ANALYSIS}

\subsection{Memory Usage}

The amount of main memory required to evaluate the face data using the proposed algorithm is known as the memory usage of the algorithm. The figure 4 shows the memory performance.

Usage of the implemented algorithms, the according to the given figure $4 \mathrm{X}$-axis contains the amount of data to be process in increasing size. Similarly the $\mathrm{Y}$ axis contains the memory usage in terms of $\mathrm{KB}$. According to the given results the memory requirement of the proposed system is higher as compared to the LDA technique.

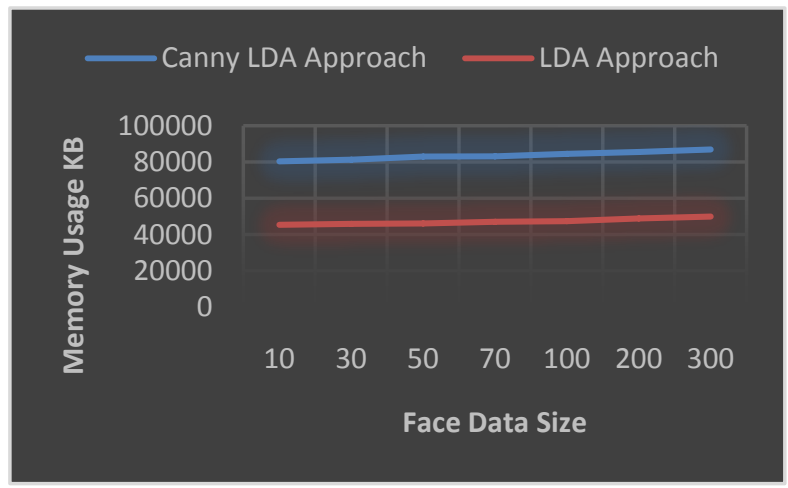

Figure 4: Memory Usage

The results show the performance of the traditional algorithm is much effective as compared to proposed in memory factor. The reason is that of this performance Canny edge detection algorithm consumes more memory to process system, so that memory is higher than of proposed system that of LDA based system.

\subsection{Time Consumption}

The amount of time required to evaluate the entire data and produces the matching of the faces, here as the time consumption of the algorithm. The utilized time of the algorithms are evaluated and given in terms of milliseconds. In this diagram the $\mathrm{Y}$ axis contains required time in $\mathrm{MS}$ and the $\mathrm{X}$ axis contains the amount of data to be processed. According to the given results the proposed method consumes less amount of time as compared to traditional approach

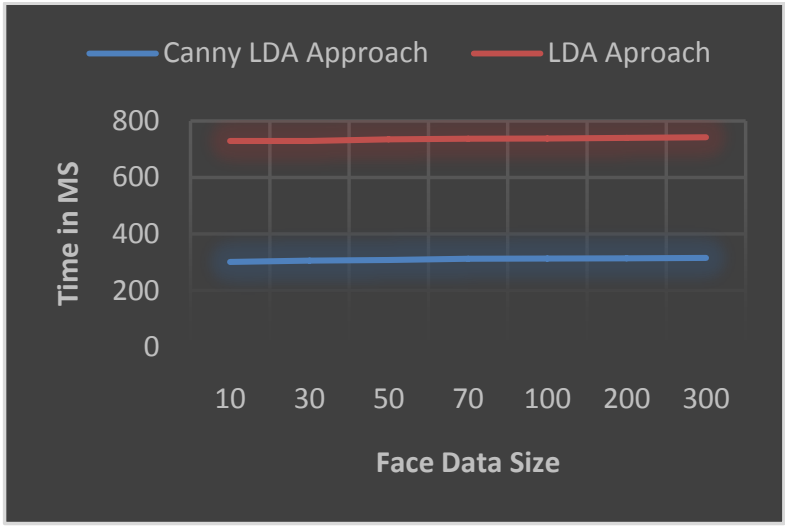

Figure 5: Time Consumption

Traditional LDA based Face recognition approach is shown in figure and table. In this diagram the $\mathrm{X}$-axis shows the implemented algorithms and the $\mathrm{Y}$ axis shows the mean time consumption of the given algorithm. According to the mean performance of the algorithms the proposed technique consumes less time as compared to the traditional approach of LDA method.

\subsection{Accuracy}

The accuracy of the algorithm provides the estimation about correctly identified prediction of images. Therefore that is an essential parameter for any data analysis algorithm. This parameter can be evaluated using the following formula.

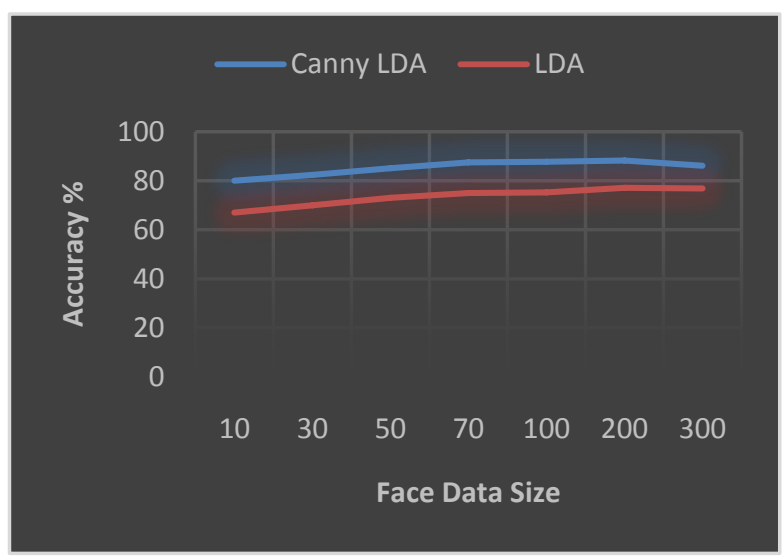

Figure 6: Accuracy

Figure 6 shows the evaluated performance in terms of accuracy. In this figure the amount of face data instances for evaluation is given in $\mathrm{X}$ axis and the $\mathrm{Y}$ axis shows the percentage accuracy obtained by the system. According to the obtained performance the accuracy of the proposed Hybrid Canny LDA face recognition algorithm is efficient as compared to the traditional LDA algorithm. The combined result over the different face data size shows the higher percentage of improvement as compared to the traditional method additionally able to produce the accuracy $80-90 \%$.

\subsection{Error Rate}

The error rate is an amount of data that is not properly recognized during the automated data analysis. That can be evaluated using the following formula: 


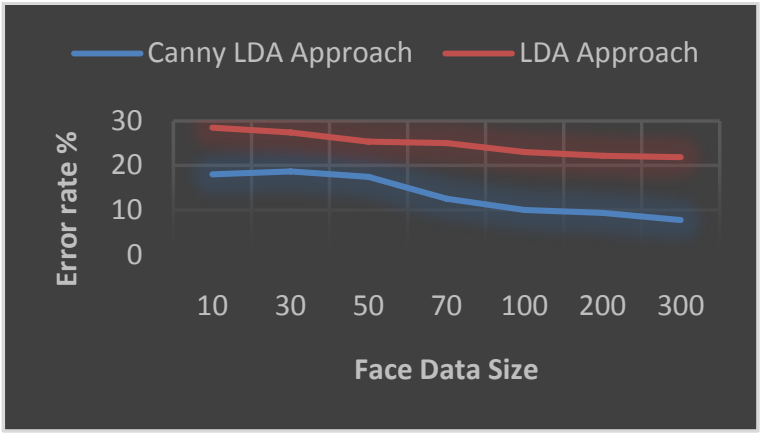

Figure 7: Error Rate

The error rate in terms of percentage of both the implemented algorithms is given using figure 7 . In this figure the size of image is given using $\mathrm{X}$ axis and the $\mathrm{Y}$ axis shows the percentage error rate. According to the given results the proposed technique produces less error rate as compared to the traditional technique of face detection. Additionally the error rates of both the systems are reducing that is a good significant with increasing size of data. In this diagram the $\mathrm{X}$ axis shows the implemented techniques and the $\mathrm{Y}$ axis shows the mean error rate percentage. According to the obtained results the proposed technique produces less error rate as compared to the traditional algorithm. Thus the proposed technique is much adoptable than classical approach.

\section{CONCLUSION}

Face recognition is a very interesting quandary. Ideally a face detection system should be able to take a new face and return a name identifying that person. Mathematically, what possible approach would be robust and fairly computationally economical? If we have a database of people, every face has special features that define that person Face recognition is a technology just reaching sufficient maturity for it to experience a rapid growth in its practical applications. Optimal feature selection (FS) is to find the minimal subset of features from original feature set that can represent the whole dataset. In this paper, a novel LDA and Canny Edge Filter based Face Recognition algorithm, HFDA, i.e. Hybrid Face Detection Algorithm is proposed. In this domain the training and testing are the two phases for pattern learn from the past experience. These patterns are used with the computational model to prepare a data model by which the target objectives are accomplished. These techniques are frequently used in the new generation for optimizing the experience of human life.

\section{REFERENCES}

[1] Rein-Lien Hsu, "Face Detection and Modeling for Recognition," PhD thesis, Department of Computer Science \& Engineering, Michigan State University, USA, 2002.

[2] Zdene` $\mathrm{k} \mathrm{R}^{\curlyvee}$ íha and Václav Matyáš, "Biometric Authentication Systems", Faculty of Informatics , Masaryk University, FI MU Report Series, November 2000.
[3] "Biometrics Overview", National Science and Technology Council, available online: http://www.biometricscatalog.org/NSTCSubcommittee/ Documents/Biometrics\%20Overview.pdf

[4] Sanjeev Kumar and Harpreet Kaur, "Face Recognition Techniques: Classification and Comparisons", International Journal of Information Technology and Knowledge Management July-December 2012, Volume 5, No. 2, pp. 361-363

[5] Rabab M. Ramadan and Rehab F. Abdel - Kader, "Face Recognition Using Particle Swarm Optimization-Based Selected Features", International Journal of Signal Processing, Image Processing and Pattern Recognition, Volume 2, No. 2, June 2009.

[6] V. Vijayakumari, "Face Recognition Techniques: A Survey", World Journal of Computer Application and Technology 1(2): 41-50, 2013

[7] B. Maison,C. Neti, and A. Senior, Audio-visual Speaker Recognition for Video Broadcast News: Some Fusion Techniques, In Multi-media Signal Processing, 1999.

[8] Wilson, Phillip Ian, and John Fernandez, "Facial feature detection using Haar classifiers." Journal of Computing Sciences in Colleges 21.4 (2006): 127-133.

[9] Scheenstra, Alize, Arnout Ruifrok, and Remco C Veltkamp, "A survey of 3D face recognition methods", Audio-and Video-Based Biometric Person Authentication, Springer Berlin Heidelberg, (2005).

[10] Ghimire, Deepak, and Joonwhoan Lee, "A robust face detection method based on skin color and edges" Journal of Information Processing Systems 9.1, PP. 141-156, 2013

[11] Pinaki Pratim Acharjya, Ritaban Das \& Dibyendu Ghoshal "Study and Comparison of Different Edge Detectors for Image Segmentation", Global Journal of Computer Science and Technology Graphics \& Vision, Volume 12 Issue 13 Version 1.0, 2012

[12] M Sudarshan, P Ganga Mohan and Suryakanth V Gangashetty "Optimized Edge Detection Algorithm for Face Recognition".

[13] Sharifi, Mohsen, Mahmood Fathy, and Maryam Tayefeh Mahmoudi. "A classified and comparative study of edge detection algorithms." Information Technology: Coding and Computing, 2002. Proceedings. International Conference on. IEEE, 2002.

[14] Poonam S. Deokar, "Implementation of Canny Edge Detector Algorithm using FPGA", International Journal of Innovative Science, Engineering \& Technology, Vol. 2 Issue 6, June 2015. 